REVISTA MATEMATICA de la

Universidad Complutense de Madrid

Volumen 1, números $1,2,3 ; 1988$.

http://dx.doi.org/10.5209/rev_REMA.1988.v1.n1.18175

\title{
Multiplicative functionals on function algebras
}

\author{
J. Gómez and J. G. Llavona ${ }^{1}$
}

ABSTRACT. Let $X$ be a completely regular Hausdorff space and $C(X)$ the algebra of all continuous $\mathbb{K}$-valued functions on $X(\mathbb{K}=\mathbb{R} \circ \mathbb{C})$. If $A \subseteq C(X)$ is a subalgebra, in [4] can be found conditions on $A$ under which each character of $A$, i.e., each non-zero $\mathbb{K}$-linear multiplicative functional $\phi: A \rightarrow \mathbb{K}$, is given by a point evaluation at some point of $X$.

In this paper we present a «Michael» type theorem for the particular case in which $X$ is a real Banach space. As consequence it is showed that if $E$ is a separable Banach space or $E$ is the topological dual space of a separable Banach space and $A$ is the algebra of all real analytic or the algebra of all real $C^{m}$-functions, $m=0,1, \ldots, \infty$, on $E$, then every character $\phi$ of $A$ is a point evaluation at some point of $E$.

Let $E$ be a real Banach space with topological dual $E^{\prime}$ and let $C(E)$ be the algebra of all continuous $\mathbb{R}$-valued functions on $E$. Let $l^{1}(\mathbb{N})=\left\{\alpha=\left(\alpha_{n}\right) \in \mathbb{R}^{N}\right.$. $\left.: \sum_{n=1}^{\infty}\left|\alpha_{n}\right|<\infty\right\}$

Theorem 1. Assume that there exists $\left(\phi_{n}\right)_{n=1}^{\infty} \subset E^{\prime},\left\|\phi_{n}\right\| \leqslant 1$ for every $n \in \mathbb{N}$, such that $\left(\phi_{n}\right)$ separates points of $E$. Let $A \subseteq C(E)$ be a subalgebra with $1 \in A$. Asstume:

(i) If $f \in A, f(x) \neq 0$ for all $x \in E$, then $1 / f \in A$.

(ii) $E^{\prime} \subset A$ and for every $\alpha=\left(\alpha_{n}\right) \in l^{1}(\mathbb{N})$, the function $\sum_{n=1}^{\infty} \alpha_{n} \cdot \phi_{n}^{2}$ belongs to $A$.

Then every character $\phi: A \rightarrow \mathbb{R}$, such that $\phi\left(\phi_{n}\right)=\phi_{n}(a)$ for every $n \in \mathbb{N}$ and some $a \in E$, is the point evaluation at $a$.

\footnotetext{
${ }^{1}$ Research partially supported by CAICYT grant PR83-2197.

1980 Mathematics Subject Classification (1985 revision): 46 E25.

Editoria] de la Universidad Complutense. Madrid, 1988.
} 
Proof. Let $\alpha=\left(\alpha_{n}\right) \in l^{1}(\mathbb{N})$ with $\alpha_{n}>0$ for all $n \in \mathbb{N}$. Condition (ii) implies that the functions:

$$
f(x)=\sum_{n=1}^{\infty} \alpha_{n} \phi_{n}^{2}(x-a) \text { and } g(x)=\sum_{n=1}^{\infty} \frac{\alpha_{n}}{n} \phi_{n}^{2}(x-a) \text { belong to } A
$$

For each $N \in \mathbb{N}$, let $x \in E$ such that $\phi(f)=f(x) ; \phi(g)=g(x)$ and $\phi\left(\phi_{i}\right)=\phi_{i}(x)$, $i=1, \ldots, N$ (a such $x$ exists after condition (i)). For this $x \in E$, we have

$$
\phi(f)=\sum_{N+1}^{\infty} \alpha_{n} \phi_{n}^{2}(x-a) ; \phi(g)=\sum_{N+1}^{\infty} \frac{\alpha_{n}}{n} \phi_{n}^{2}(x-a)
$$

Therefore $0 \leqslant N \phi(g) \leqslant \phi(f)$ and it follows that $\phi(g)=0$.

If $h \in A$ is given, let $y \in E$ such that $\phi(h)=h(y)$ and $\phi(g)=g(y)$. Since $\phi(g)=g(y)=0$, it follows that $\phi_{n}(y)=\phi_{n}(a)$ for all $n \in \mathbb{N}$, i.e., $y=a$ and $\phi(h)=h(a)$.

Remark 1. The hypothesis on the real Banach space $E$ in Theorem 1 is equivalent to say that $E^{\prime}$ is $\sigma\left(E^{\prime} ; E\right)$-separable. Therefore it holds when $E$ is a separable Banach space and when $E$ is the topological dual space of a separable Banach space.

\section{Consequences}

Let $A(E)$ be, respectively $C^{m}(E)(m=0,1, \ldots, \infty)$, the subalgebra of $C(E)$ of all real analytic functions (see [2]), respectively of all $C^{m}$-functions in the Fréchet sense, on $E$.

Corollary 1. If $E$ is finite dimensional and $A=A(E)$ or $A=C^{m}(E)$, then every character $\phi: A \rightarrow \mathbb{R}$ is a point evaluation at some point of $E$.

Proof. This follows from Theorem 1 if we consider $\left(\phi_{n}\right)$ as the canonical projections.

Proposition 1. For every character $\phi: A(E) \rightarrow \mathbb{R}$, the restriction $\phi_{\mid E^{\prime}}$ is $\sigma\left(E^{\prime} ; E\right)$ sequentially continuous.

Proof. Assume that $\left(x_{n}^{\prime}\right) \subset E^{\prime}$ converges to zero for the $\sigma\left(E^{\prime} ; E\right)$-topology. If $\phi\left(x_{n}^{\prime}\right) \neq 0$, there are $\alpha>0$ and $\left(x_{n_{p}}^{\prime}\right)$, subsequence of $\left(x_{n}^{\prime}\right)$, such that

$$
\left[\phi\left[\frac{x_{n_{p}}^{\prime}}{\sqrt{\alpha}}\right]\right]^{2}>1
$$

for every $p \in \mathbb{N}$. Since $\left(x_{n_{p}}^{\prime}\right) \rightarrow 0(p \rightarrow \infty)$ for the $\sigma\left(E^{\prime} ; E\right)$-topology, 
the function

$$
f(x)=\sum_{p=1}^{\infty}\left[\frac{x_{n p}^{\prime}(x)}{\sqrt{\alpha}}\right]^{2 p}
$$

is well defined and $f \in A(E)$. (See ([2], Th. 6)). For each $N \in \mathbb{N}$,

$$
\phi(f) \geqslant \phi\left[\sum_{p=1}^{N}\left[\frac{x_{n_{p}}^{\prime}}{\sqrt{\alpha}}\right]^{2 p}\right]=\sum_{p=1}^{N}\left[\phi\left[\frac{x_{n_{p}}^{\prime}}{\sqrt{\alpha}}\right]\right]^{2 p}
$$

Therefore $\sum_{p=1}^{\infty}\left[\phi\left[\frac{x_{n_{p}}^{\prime}}{\sqrt{\alpha}}\right]\right]^{2 p}<\infty$ and then $\left[\phi\left[\frac{x_{n_{p}}^{\prime}}{\sqrt{\alpha}}\right]\right]^{2 p} \rightarrow 0(p \rightarrow \infty)$, which is a contradiction because $\left[\phi\left[\frac{x_{n_{p}}^{\prime}}{\sqrt{\alpha}}\right]\right]^{2}>1$ for all $p \in \mathbb{N}$.

Corollary 2. Let $E$ be a separable Banach space and $\phi: A(E) \rightarrow \mathbb{R}$ a character. Then $\phi_{E^{\prime}}$ is a point evaluation at some point of $E$.

Proof. This is immediate from Prop. 1, since by ([5], Ch. IV; Th. 6.2 and Corollary 3) for $\phi_{\mid E^{\prime}}$ to be $\sigma\left(E^{\prime} ; E\right)$-continuous it suffices to show that $\phi_{\mid E^{\prime}}$ is $\sigma\left(E^{\prime} ; E\right)$-sequentiaily continuous.

Corollary 3. Let $E$ be a separable Banach space and $\phi: A(E) \rightarrow \mathbb{R}$ a character. Then $\phi$ is a point evaluation at some point of $E$.

Proof. This is immediate from Theorem 1, Remark 1 and Corollary 2.

Let $F$ be a separable Banach space and $\left(y_{n}\right)_{n=1}^{\infty}$ a dense subset in $\{y \in F:\|y\| \leqslant 1\}$. Let $E=F^{\prime}$. Let $\phi_{n}: \mathrm{E} \rightarrow \mathbb{R}$ be defined as $\phi_{n}(x)=x\left(y_{n}\right)$. Then $\phi_{n} \in E^{\prime},\left\|\phi_{n}\right\| \leqslant 1$ and $\left(\phi_{n}\right)_{n=1}^{\infty}$ separates points of $E$. The mapping $y \rightarrow \phi_{y}$, defined as $\phi_{y}(x)=x(y)$, allow us identify $F$ with a subspace of $E^{\prime}=F^{\prime \prime}$. Thus, if $\phi: A(E) \rightarrow \mathbb{R}$ is a character, Prop. 1 implies that $\phi_{\mathrm{F}}$ is $\|\cdot\|$-continuous, therefore $\phi_{\dot{F}} \in F^{\prime}=E$. Then, it follows that there exists $a \in E$ such that $\phi\left(\phi_{n}\right)=\phi_{n}(a)$ for all $n \in \mathbb{N}$. Now the following Corollary is clear after Theorem 1.

Corollary 4. Let $E$ be a topological dual space of a separable Banach space and $\phi: A(E) \rightarrow \mathbb{R}$ a character. Then $\phi$ is a point evaluation at some point of $E$.

Corollary 5. Assume that $E$ is a separable Banach space or $E$ is the topological dual space of a separable Banach space. Then every character $\phi: C^{m}(E) \rightarrow \mathbb{R}, m=0,1, \ldots, \infty$, is a point evaluation at some point of $E$. 
Proof. $\phi_{|A| E \mid}$ is a point evaluation by Corollary 3 and Corollary 4 . Thus, $\phi$ satisfies conditions of Theorem 1 with $A=C^{m}(E)$.

Remark 2. The Corollary 5, for the particular case $E$ a separable Banach space and $m=\infty$, can be found in [1]. Also, for $E$ with $C^{m}$-partitions of unity and $m<\infty$, see [3]

\section{References}

[1] ARIAS-DE-REYNA, J. «Real Valued Homomorphisms on Algebras of Differentiable Functions», (preprint).

[2] BochNaK, J. "Analytic Functions in Banach Spaces». Studia Math. T. $X X X V$ (1970), 273-292.

[3] JaRAmillo, J. A. «Topologies and Homomorphisms on Algebras of Differentiable Functions». (Preprint).

[4] Michael, E. A. «Locally Multiplicatively-Convex Topological Algebras». Memoirs of the A.M.S., number 11 (1952).

[5] SCHAEFER, H. H. «Topological Vector Spaces». Graduate Texts in Math. SpringerVerlag (1971).

Departamento de Análisis Matemático

Facultad de CC. Matemáticas

Recjbido: 12 de febrero de 1988

Universidad Complutense

28040 Madrid 